\title{
A Readout Unit for High Rate Applications
}

\author{
José Toledo, François Bal, David Domínguez, Angel Guirao, Hans Müller
}

\begin{abstract}
The LHCb Readout Unit (RU) is a custom entry stage to the readout network of a data acquisition or trigger system. It performs sub-event building from multiple link inputs towards a readout network, via a PCI network interface or alternatively towards a high-speed link, via a S-link interface. Incoming event fragments are de-randomized, buffered and assembled into single sub-events. This process is based on a lowoverhead framing convention and matching of equal event numbers. Programmable logic is used both in the input and output stages of the RU module which may be configured either as data link multiplexer, or as entry stage to a readout or trigger network. All FPGAs are interconnected via the PCI bus, which is hosted by a networked microprocessor card. It's main tasks are remote FPGA configuration and initialization of the PCI cards. The RU hardware architecture has been optimized for a throughput of up to $200 \mathrm{Mbyte} / \mathrm{s}$ at a $1 \mathrm{MHz}$ trigger rate, as required by the most demanding application, the LHCb level-1 trigger network. A custom traffic-scheduling link is available for applications like pipelined destination address allocation.
\end{abstract}

\section{INTRODUCTION}

$\mathrm{T}^{\mathrm{R}}$ RIGGER and DAQ systems of the LHCb experiment [1] share the need for Readout Units that receive incoming event fragments (EF) on multiple data links and assemble them into larger sub-events (SE's) as a pre-stage of the full event-building. Both EF's and SE's conform to a lowoverhead Sub-event Transport Format (STF) designed for the RU. The RU's main operation is to re-assemble EF's or SE's that belong to the same event identifier into new SE's, buffer and verify them and retransmit them at the output in the same order as they were received. Two possibilities for interfacing exist at the output: PCI or Slink [2]. An output link may again feed the input of another RU. The repeatable sub-event assembly process for programmable logic is developed and simulated using the IEEE-1076 VHDL standard language for unproblematic portability and maintenance. For highest data

Manuscript received June 8, 2001; revised November 12, 2001. This work was performed as part of the computing project of the LHCb experiment at CERN.

José Toledo, designer of the Readout Unit is with the Electronics Engineering Department, Polytechnic University of Valencia, Spain (telephone:+34-962-849300, e-mail: jtoledo@eln.upv.es).

Francois Bal is with CERN EP Division, Geneva, Switzerland (e-mail: Francois.Bal@cern.ch).

David Dominguez was with CERN EP Division, Geneva, Switzerland (email: David.Dominguez@cern.ch).

Angel Guirao is with CERN EP Division, Geneva, Switzerland as doctoral student (telephone: +41-22-7679761, e-mail: Angel Guirao@cern.ch).

Hans Müller is project leader of the Readout Unit Project in the CERN EP-ED group, Geneva, Switzerland (e-mail: Hans.Muller@cern.ch). transmission performance via the PCI bus, Field Programmable System Chips ${ }^{1}$ (FPSC) which contain 64 bit PCI cores are used.

Three areas have been identified in the LHCb experiment where sub event-building functionality is needed (Fig. 1):

1. Front End link Multiplexers (FEM) after the Level-1 buffers

2. Readout Unit (RU) entry stage for the Data Acquisition system (DAQ) [3]

3. Readout Unit (RU) entry stage to the Level-1 Topology Trigger network [4] (Fig. 3)

\section{A. FEM and DAQ applications}

Front-end multiplexers reduce the number of low-bandwidth data links originating from the level-1 buffers. Up to 16 links can be grouped together on the inputs of one RU whose output stage is re-transmitting to a single link of correspondingly higher bandwidth. The purpose is to adapt the bandwidth to the input capacity of the next stage and reduce the high number of links.

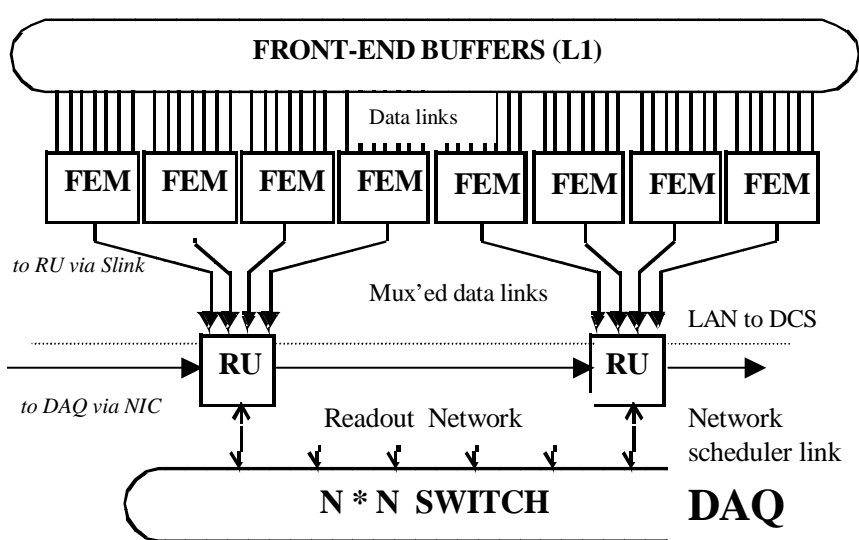

Fig. 1. The Readout Unit can be used for both Front End Multiplexer (FEM) and genuine Readout Unit (RU) applications. The DAQ system of $\mathrm{LHCb}$ requires that an average $4 \mathrm{Gbyte} / \mathrm{s}$ bandwidth (100 Kbyte events at 40 $\mathrm{KHz}$ rate) are transferred via a layer of RU's which interface to a N-by-N switch, where $\mathrm{N}$ is in the order of 100 but may scale with the final trigger rate. A LAN connection on each RU provides remote control and monitoring. A 16bit network scheduler link, interconnecting RU's horizontally is required in high rate trigger applications.

The bandwidth requirement for a FEM is a factor of four less than for a RU in the DAQ entry stage since four FEM output links feed one RU input. Both FEM and RU are the same hardware modules, programmed differently and performing

${ }^{1}$ FPSC is a term for FPGA chips with embedded system cores of Lucent Technologies 
sub-event building in two successive stages. Their main parameters are summarized in table I.

A Readout Unit [5][6], configured to operate as FEM, outputs to data links via a S-link interface card. When operating as DAQ interface the RU outputs to a PCI interface, specifically to a Network Interface Cards (NIC). This implies that the output stage of the RU can perform PCI configuration of the NIC and that sub-events in the RU buffer can be transferred to the NIC via the PCI bus. For the PCI initialization, a networked processor card is resident on the PCI root segment, performing the tasks of a PCI host. The high rate data transfers between the RU buffer and a NIC make use of high bandwidth PCI bus core logic which is embedded in the programmable logic chips.

TABLE I

Front-END MULTIPLEXER AND DAQ READOUT UNIT APPLICATIONS

\begin{tabular}{lcc}
\hline \multicolumn{1}{c}{ PARAMETER } & FEM & DAQ \\
\hline Number of inputs & 1 to 16 & 1 to 4 \\
Fragment size per input & up to 1 Kbyte & up to 4 Kbyte \\
Sub-event size & $1 \mathrm{Kbyte}$ & $4 \mathrm{Kbyte}$ \\
Rate & $40 \mathrm{kHz}$ & $40 \mathrm{kHz}$ \\
Throughput & $40 \mathrm{Mbyte} / \mathrm{s}$ & $160 \mathrm{Mbyte} / \mathrm{s}$ \\
\hline
\end{tabular}

The operation of a FEM, like the one of a RU, consists in building of sub-events from multiple inputs. Complete subevents in the buffer of a FEM get immediately written to the S-link transmitter card. The FEM is a single-destination, write-through operation. The output stage in the RU application however is part of the DAQ event-building domain, i.e. the network and synchronization protocols between the sub-event buffer and the readout network are handled via a NIC card, which is configured and controlled via the PCI bus.

\section{1) Sub event Transport Format (STF)}

The 32bit STF format for transporting and building of subevents was designed for low a overhead, pipelined FPGA process. It includes parity checking of headers and trailers. Three headers and one trailer encapsulate STF sub-events (Fig. 2) at both inputs and output of a RU. Data blocks transported by STF frames are transparent to the RU logic and must hence be consistently formatted.

\begin{tabular}{|c|c|c|}
\hline \multicolumn{2}{|c|}{ DAQ Event Number } & SH \\
\hline Link ID & Checkbits & Type \\
\hline Offset to Error Block & Reserved & EB Size \\
\hline \multicolumn{3}{|c|}{ DATA BLOCK(S) } \\
\hline \multicolumn{3}{|c|}{ ERROR BLOCK (EB) } \\
\hline Status & I Size & ST \\
\hline
\end{tabular}

Fig. 2. Sub-event Transport Format STF, containing some data blocks of a sub-event and an optional error block. The event number is contained in the header and the total size in the trailer. The link ID identifies the outgoing link. SH (S-Link Header) and ST (S-Link Trailer) are 4-bit fields used by Slink control words to report parity errors. The first and last words in the STF are S-link control words which get identified in the destination logic in order to generate frame signals. The error block and the check bits are optional. The equation $\mathrm{TS}=4+\mathrm{OEB}+\mathrm{EBS}$ always allows to verify STF consistency.

The following fields are contained in the STF format:

1. DAQ Event Number: 28-bit monotonically increasing number that uniquely identifies sub-events

2. Link ID: 16-bit code identifying the link transmitter

3. Check bits: 8-bit optional field. Default is zero

4. Type: 8-bit field for a type of data (i.e. TRIGGER, DAQ, etc )

5. Offset to Error Block: 16-bit offset to the error block. Points to the trailer if EB-size is zero

6. EB Size: Error Block Size, may be zero

7. Status: 8-bit field for fast status information. Each bit represents one of eight error classes

8. Total Size: 20-bit field containing the total number of words in the sub event (including framing words)

\section{B. Trigger application (Vertex trigger network of $\mathrm{LHCb}$ )}

The Vertex trigger application requires up to $200 \mathrm{Mbyte} / \mathrm{s}$ throughput for small fragments at a nominal 1-MHz trigger rate (table II). The trigger network (Fig.3) is formed by a two-dimensional torus of $10 \mathrm{Gbit} / \mathrm{s}$ ringlets with $\mathrm{CPU}$ nodes at each crossing point [7]. Events are sent by the RUs as PCI bursts from DMA engines in the RU output stages. These feed the 64 bit NIC interfaces making use of a 'tandem master' mode, in order to achieve the required high bandwidth and rates with the very small event-sizes of the $\mathrm{Si}$ strip detectors.

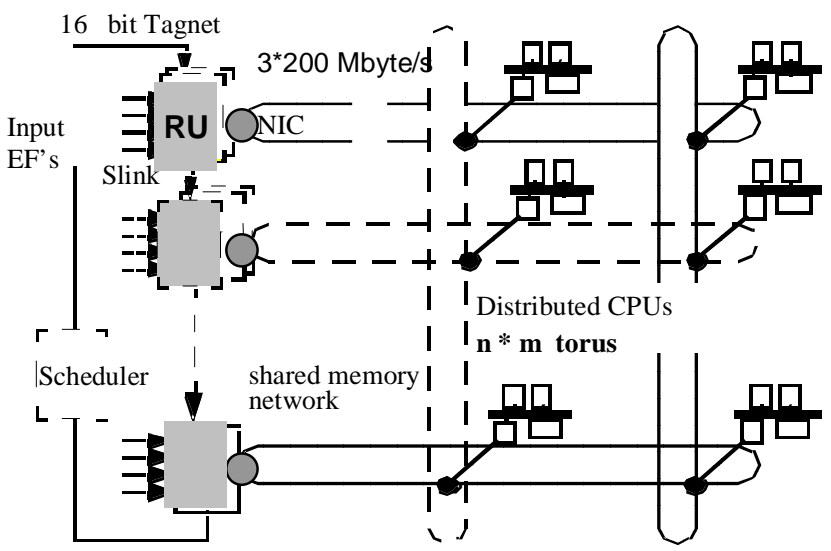

Fig. 3. Readout Unit in the 1-MHz Vertex trigger application of the $\mathrm{LHCb}$ Si Strip detector. A two-dimensional torus network with distributed CPUs in the intersections is used. The torus is implemented as horizontal and vertical SCI ringlets of $800 \mathrm{Mbyte} / \mathrm{s}$ bandwidth each. The Tagnet scheduling is used to allocate the CPU destination addresses to all RUs.. According to Table II, up to four RU's are needed to fill one ringlet. As an example, 20 RU's would be needed, for a total throughput requirement of 4 Gbyte/s in a 5-row network.

The network and the NIC card comply with the IEEE-1596 SCI standard [8], supporting $800 \mathrm{Mbyte} / \mathrm{s}$ bandwidth per ringlet. The overhead-free shared memory paradigm of SCI (direct write to destination memory at a microsecond latency) achieves transfer rates beyond $1 \mathrm{MHz}$ with small event 
fragments (typically 64 to 128 byte). A custom trafficscheduling network ("Tagnet") is used for the RU output timing and address allocation in order to achieve highest bandwidth utilization in the torus. Congestion is avoided by forbidding that more than one RU sends data to the same column at the same time. A Tagnet scheduler distributes free "address-tags" of CPUs to the RU's, which intercept and return them after use. The 16 bit wide custom 'Tagnet' protocol is implemented on the RU as programmable LVDS signals, serialized on standard RJ45 input and output connectors. Since this trigger application is very sensitive to overheads, a minimal transport format like STF is used.

TABLE II

READOUT UNIT FOR LEVEL-1 VERTEX TRIGGER APPLICATION

\begin{tabular}{ll}
\hline Number of inputs per RU & 1 to 4 \\
Fragment size per input & $\sim 100$ byte \\
Sub-event size on RU output & $\sim 200$ byte \\
Nominal trigger rate & $1 \mathrm{MHz}$ \\
Throughput (max) & $200 \mathrm{Mbyte} / \mathrm{s}$ \\
\hline
\end{tabular}

\section{THE READOUT UNIT}

In a common approach for the FEM and DAQ applications in the $\mathrm{LHCb}$ experiment, a programmable sub-event builder module was designed, taking input from up to four data Slink receivers (mezzanine slots on the right in Fig. 5) and outputting via the PCI bus to a Network Interface Card (NIC) (top left mezzanine card in Fig. 5). An S-Link card may be used in the same slot as the NIC card for low-throughput FEM applications.

The RU module was originally designed [9] for the DAQ and FEM applications requiring a maximum throughput of ca. $80 \mathrm{Mbyte} / \mathrm{s}$. In a revision it has been modified to meet also the high rate, high bandwidth requirements of the $\mathrm{LHCb}$ Level-1 Topology Trigger. The final RU architecture (Fig.4) consists of two parallel data paths with two S-link inputs each and two Sub-Event Merger (SEM) input stages. Operating in parallel, each SEM block builds sub-events in its associated sub-event buffer, implemented as Dual Port Memory (DPM).

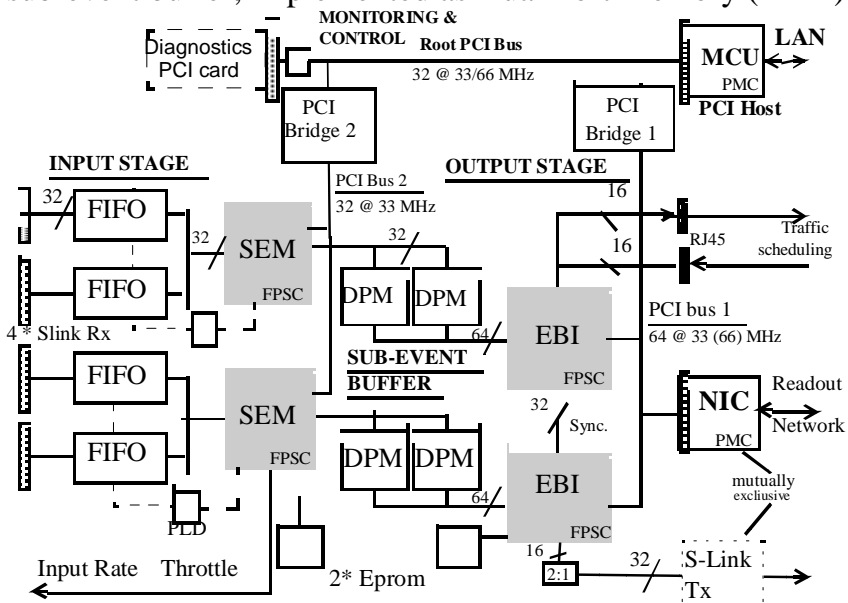

Fig. 4. Hardware architecture of the Readout Unit: two parallel Sub Event
Merger (SEM) stages merge incoming fragments (by comparing event numbers) into their associated DPM buffers. The dual Event Builder Interface (EBI) logic combines the two DPM buffers to generate and transmit single sub-events to S-link or PCI. The networked Monitoring and Control Unit (MCU) is a PMC card functioning as host CPU of the root PCI segment. A decoupled, 64 bit PCI secondary bus doubles in function as the output bus of the EBI and as configuration bus of the EBI and NIC. Another decoupled 32 bit PCI bus provides access to control registers in the SEM. An auxiliary PCI connector on the root segment is available for diagnostics with standard PCI cards.

The sub-event building process is based on counting of a fixed number of received input fragments per event (implying that each channels sends one STF frame per event) and on matching of their event-numbers that increase monotonically for each new event.

The Event Builder Interface (EBI) logic in the output stage combines the sub-event fragments from the two DPM buffers of the input stage into a single sub-event. Depending on the application the EBI transmits either via the 64 bit PCI output bus to a NIC card or via a $16 / 32$ bit bus to an S-link card. For synchronization, the two EBI logic blocks make use of a synchronization bus which provides that new sub-events can get transmitted within a single PCI bust to the NIC ( or as FIFO dump to S-link). A throttle signal is generated whenever the fill-states of the buffers become critical.

A monitoring and control unit (MCU) card was designed [10] for remote control on the RU via a $10 / 100 \mathrm{Mb} / \mathrm{s}$ LAN connection to the Detector Control System (DCS) [11].

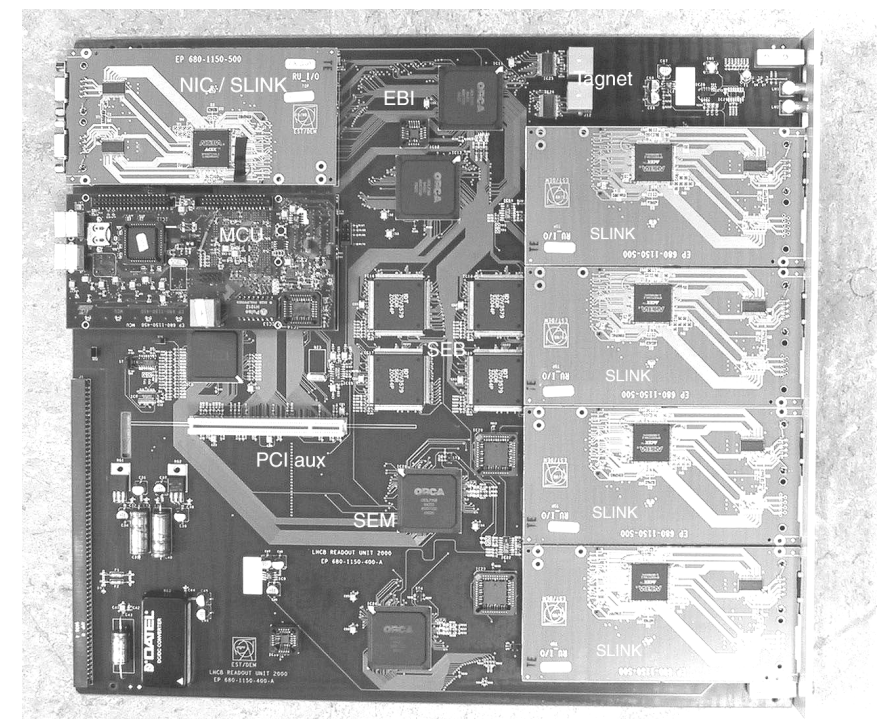

Fig. 5 Photo of the Readout Unit equipped with 6 mezzanine PMC cards. The 9U IEEE-960 crate mechanics is used as framework for power, mechanics and cooling; the back-plane bus is unused. All four S-link data input cards are at the front-panel (right side).A pair of FPSC chips (ball grid array packages) contain the programmable logic of the SEM input stage and another pair the logic of the EBI output stage. Both are visible on bottom and top, centered by four DPM memory chips, which form the SEB data buffer. The interface cards for the readout network (PMC card, left top) can be either a S-Link or a NIC card. The networked MCU processor is a PMC (IEEE P-1386) card, situated below the NIC/SLINK card. A PCI analyzer may be used in the PCI auxiliary connector for RU diagnostics (horizontal PCI connector)

The tasks of the MCU card (PCI host) are:

1. Monitoring of the sub-event buffer via direct access 
2. Error handling and reporting

3. Remote control of the operating parameters of the RU

4. PCI bus initialization (bridges, FPSC chips, NIC card)

5. Downloading of FPSC configuration bitmaps

6. Control and monitoring of SEM/EBI status registers

7. Configuration of high-level NIC protocols

8. Emulation of RU functions before implementation

The Linux operating system of the MCU configures the I/O registers and memory space associated with each logic block via the standard 256 byte PCI configuration space, embedded in the PCI cores of each FPSC chip. The SEB buffers are mapped under Linux into the memory space of the MCU from where they can be accessed by user routines [10].

\section{A. Input stage}

Up-to four S-link receiver cards write incoming fragments into the RU's receiver FIFOs from where the Sub-Event Merger logic (SEM) reads them when fully received. Each SEM identifies those fragments that share the same event number and writes combined sub-event block to it's output buffer together with a pointer to its location, stored in a separate directory section of the buffer.

\section{B. Sub-event buffer (SEB)}

The two output buffers of the input stage compose the RU's Sub-Event Buffer (SEB), implemented as dual port memory between input and output stage (Fig. 4). Most of its capacity ${ }^{2}$ is used for storing sub-event data; only a small part is used as a pointer directory. Each of the two SEB banks consists of 32-bit-wide memories connected for 32-bit access from the SEM and 64-bit access from the EBI side. The fill state of the SEB is monitored by programmable logic, which returns a 'trigger throttle' signal via a front-panel signal backwards to the trigger supervisor.

\section{Output stage}

The EBI logic in the output stage scans the directory in the SEB buffer to identify the locations of sub-events in the two SEB buffers that belong together. Using simple STF rules (Fig. 6), the EBI logic generates the sub-event format of the new, combined sub-event. The 64 bit PCI bus is used to generate a single, STF-framed sub-event burst for the Network Interface Card (NIC). There are two possible subevent transfer protocols between the EBI logic and the NIC:

1. A "Push" protocol, in which the dual EBI logic reads, buffers and forwards sub-event blocks, as PCI initiator into the memory area of the NIC. Sub-event building, i.e. formatting of a single SE, is performed "on the fly". Such 'source initiated' transfers are controlled by "DMA write engines" within the EBI which only need a minimal synchronization protocol between NIC and EBI logic.

2. A "Pull" protocol where a "DMA read engine" is implemented in the NIC card for mastering of PCI read bursts. The NIC card can directly access sub-event data in the SEB buffers ( via PCI memory mapping of the
SEB buffers through the EBI). The assembly of fragments into single sub-events and their transmission is part of an 'intelligent' NIC card logic. This is slower, however simplifies the EBI logic to target-only operation and allows the full event-building protocol to be handled exclusively by the NIC card.

3. A third (slow) possibility exists for application development, where the MCU implements all, or part, of the sub-event building process in software.

An example of a sub-event building from four STF formatted fragments into a new sub-event is depicted in Fig.6

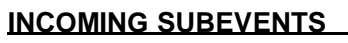

A

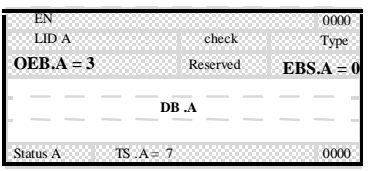

\section{NEW SUBEVENT}

$\mathrm{OEB}=$ offset to error block $=$ sizeof $(A+B+C+D)$

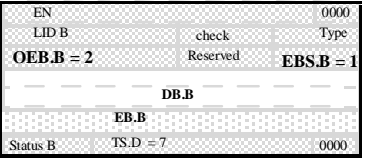

C

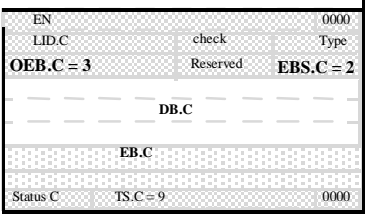

D
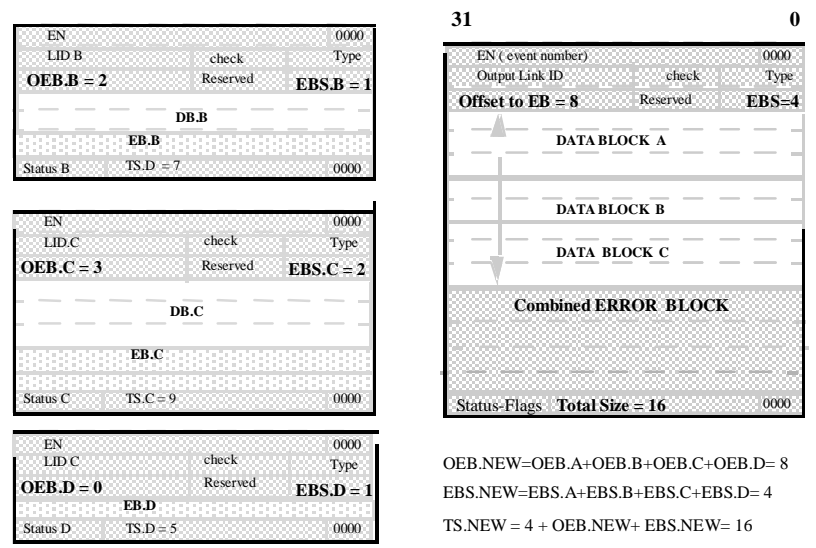

OEB.NEW=OEB. $\cdot$ +OEEB.B $B+O E B \cdot C+O E B \cdot D=8$ EBS.NEW $=$ EBS. $. A+E B S . B+E B S . C+E B S . D=4$ TS.NEW $=4+$ OEB.NEW + EBS.NEW $=16$

Fig. 6. Example of a sub-event construction from four fragments, one from each input link. Fragment A contains no error block, D is empty. The new sub-event has the same event number (EN) as the input fragments. The Link ID of the new sub-event is output link identifier. The offset to error block and the error block size result from adding the data block or error block sizes of A, B, C and D, respectively. The new data and error blocks are the concatenation of the input data and error blocks. The status byte is the logic OR operation of the four incoming status bytes. The total size (TS), offset to error block (OEB) and the error block size (EBS) is recalculated for the new sub-event according to the simple rules shown.

\section{INPUT STAGE PERFORMANCE}

An analytical expression (1) determines the maximum trigger rate $\left(f_{\text {trigger }}\right)$ for a given event-fragment size $(S)$ expressed in bytes, according to the input stage algorithm currently implemented in the SEM [4]. The input stage consists of two identical and parallel data paths. For each data path, the time needed to process an event $\left(t_{\text {event }}\right)$ equals to twice the number of clock periods required to read an EF from a FIFO via a 32-bit bus plus an extra cycle for the firstword latency. The FIFO turnaround cycles overlap with the first-word latency.

$$
\begin{aligned}
& t_{\text {event }}=2 \cdot(1+S / 4) \cdot T_{c l k}=(4+S) \cdot T_{c l k} / 2 \\
& f_{\text {trigger }}=1 / t_{\text {event }}=\frac{2 \cdot f_{c l k}}{4+S}
\end{aligned}
$$

${ }^{2} 0.5$ to 2 Mbyte is the total SEB capacity 
The FPSC-specific tools ${ }^{3}$ for placement \& routing report achievable clock frequencies of the input stage ${ }^{4}$ in excess of $60 \mathrm{MHz}$. The maximum trigger frequency for nominal $\mathrm{EF}$ sizes for each application is shown in table III.

\section{OutPut Stage PERformance}

\section{A. Multiplexer performance (quad Slink to single Slink)}

The performance is limited by the FPSC clock frequency and a 16-bit wide output ${ }^{5}$ bus for S-link. A first, nonoptimized implementation reached $45 \mathrm{MHz}$ clock frequency, corresponding to $90 \mathrm{Mbyte} / \mathrm{s}$ raw bandwidth to the S-Link output. The first test algorithms in the EBI consumes 35 cycles for synchronization and header/trailer generation, 8 cycles to send header and trailer to S-link and 512 cycles to send the sub-event payload to S-link (1 Kbyte). This makes a total of 555 cycles per event, or $81 \mathrm{kHz}$ maximum trigger rate with $45-\mathrm{MHz}$ FPSC operation. With an optimized $55-\mathrm{MHz}$ operation, we expect a rate limit for the multiplexer application around $100 \mathrm{kHz}$. The maximum rate for an arbitrary sub-event size $(S)$ is given by (2).

$$
f_{\text {trigger }}=1 / t_{\text {event }}=\frac{f_{\text {clk }}}{35+S / 2}
$$

\section{B. DAQ performance (quad S-link to NIC)}

Both a "push" and a "pull" protocol can be implemented. The read performance (pull) via PCI is known to be slow (hence high-performance PCI cards use source-initiated DMA transfers, i.e. pushing).

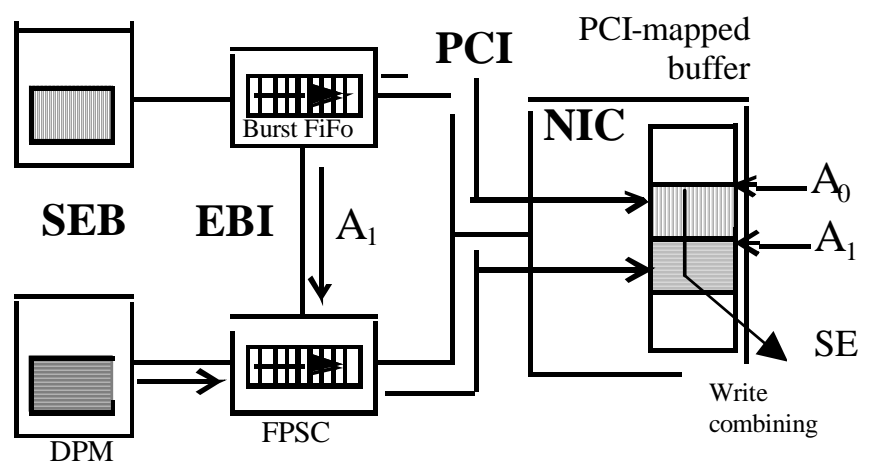

Fig. 7. Tandem operation of the two EBI FPSCs as PCI masters. One EBI fills its internal burst FIFO $^{6}$ with data while the second FPGA is flushing its FIFO to the PCI bus. In order to write a contiguous SE into the NIC memory, the FPSC that writes the first part of the sub-event gives a pointer to the other FPSC indicating the next free address in the NIC PCI-mapped memory $\left(\mathrm{A}_{1} \text { in the figure }\right)^{7}$.

\footnotetext{
${ }^{3}$ Lucent Technologies' Orca Foundry software

${ }^{4}$ All FPGAs in the RU are Lucent Technologies' Orca 3LP26BA352. See http://www.lucent.com/micro/fpga

${ }^{5}$ This 16-bit bus is demultiplexed to match the 32-bit S-Link connector

6 Internal PCI FIFO in Lucent Technologies' OR3LP26B FPSCs are 256byte deep, approx. twice the nominal size of the sub-event block read from the SEB

${ }^{7}$ Contiguous sub-events are automatically generated if the NIC supports write combining (the two transactions are merged by a PCI bridge)
}

To achieve the nominal $40 \mathrm{kHz}$ level-1 rate, the all-inclusive PCI burst per output sub-event must not last more than 25 us, which is easily achieved for a nominal 4 Kbyte sub-event with DMA engines and a 64 bit PCI bus. However a fast readout network technology, like Gigabit Ethernet limits the NIC's output performance to a maximum trigger rate of 30 $\mathrm{kHz}$, since at least $32.8 \mu$ s have been measured to transmit 4Kbyte sub-events. This limits the event-sizes for Gigabit technologies in the $40 \mathrm{kHz}$ DAQ application to ca. 3 Kbyte. Further performance penalties may apply if specific NIC cards are not 64 bit capable.

\section{VELO trigger input performance (quad S-link to NIC)}

A full "push" protocol is used in this application. No wait states are introduced by the FPSC but NIC's in SCI technology add three wait states when responding to a target write. For a typical 64-byte payload, each EBI transfers 96 bytes (twelve 64-bit PCI data words) to the NIC. One extra PCI data phase is added by each FPSC for the frame. The successive write operations get synchronized via the 32-bit bus interconnecting the two FPSCs (Fig. 7).

The minimum number of cycles required to transfer a complete sub-event to the NIC is 18 cycles per FPSC (13 data phases, 3 cycles for NIC, 1 address and 1 PCI turnaround cycle), resulting in a total of 36 cycles per subevent, meaning that FPSC and PCI clock frequencies beyond $36 \mathrm{MHz}$ are required to achieve a $1-\mathrm{MHz}$ rate. The PCI buses (see Fig. 4) will be operated at $50-66 \mathrm{MHz}^{8}$ to achieve 1.3 $\mathrm{MHz}$ as a maximum trigger rate for 200-byte sub-events. First measurements with tandem FPSC masters like in the RU reporte a $1.12 \mathrm{MHz}$ operation [7]. In general, the maximum trigger rate for a given sub-event size of $S$ byte and PCI frequency $f_{c l k}$ is given by (4).

$$
f_{\text {trigger }}=\frac{f_{c l k}}{12+S / 2}
$$

\section{CONCLUSIONS}

Table III compares the nominal requirements with the RU performance for the three target applications. In the least demanding application (FEM) the output stage limits the trigger rate to $100 \mathrm{KHz}$, assuming a 55-MHz FPSC operation.

The DAQ performance is limited by the standard Gigabit technologies (Gigabit Ethernet: bandwidth 128 Mbyte/s, practically achievable ca. $50 \mathrm{Mbyte} / \mathrm{s}$ ), resulting in a larger number of RUs and a larger switch when compared to 10 Gbit technologies like used in the VELO-L1 trigger application.

The use of "push" protocols, tandem PCI master operation and 64 bit, $66 \mathrm{MHz}$ PCI bus operation for a 10 Gbit NIC network interface achieves the $1 \mathrm{MHz}$ performances with a throughput of up to $1 / 4 \quad \mathrm{Gbyte} / \mathrm{s}$ as required for the trigger application.

\footnotetext{
8 Any frequency in the range $33-66 \mathrm{MHz}$ is considered by the PCI specification as 66-MHz mode PCI
} 
TABLE III

READOUT UNIT PERFORMANCE

\begin{tabular}{lccc}
\hline & FEM & DAQ & VELO \\
\hline Sub-event size & $1 \mathrm{Kbyte}$ & $3 \mathrm{Kbyte}$ & $\sim 200 \mathrm{byte}$ \\
Nominal rate & $40 \mathrm{kHz}$ & $40 \mathrm{kHz}$ & $1 \mathrm{MHz}$ \\
Input stage rate & $460 \mathrm{kHz}$ & $117 \mathrm{kHz}$ & $2 \mathrm{MHz}$ \\
$\begin{array}{l}\text { Output stage rate } \\
\text { Max. achievable }\end{array}$ & $100 \mathrm{kHz}$ & $40 \mathrm{kHz}$ & $1.3 \mathrm{MHz}$ \\
throughput & 100 & $120 \mathrm{Mbyte} / \mathrm{s}$ & $270 \mathrm{Mbyte} / \mathrm{s}$ \\
\hline
\end{tabular}

\section{STATUS AND OUTLOOK}

The Readout Unit project is a joint activity of the CERN EP-ED electronics group and the LHCb experiment. A first prototype was built in '2000, which led to a revised Readout Unit in early 2001. The VHDL code optimization for the 100 $\mathrm{kHz}$ FEM multiplexer application are being worked on. The DAQ performance depends on the readout network technology choice. For the $1 \mathrm{MHz}$ VELO topology trigger application, an already tested application [7] with tandem PCI masters and an SCI network will be ported to the RU.

\section{REFERENCES}

[1] LHCb Technical Proposal, CERN/LHCC 98-4, chapt. 13,

[2] S-Link, for information see the Slink homepage URL http://www.cern.ch/HSI/s-link/

[3] J.-P.Dufey, M.Frank, F.Harris, J.Harvey, B.Jost, P.Mato, H.Müller, "The LHCb Trigger and Data Acquisition System", IEEE Trans. Nucl. Sci, vol 47, no 2, pp. 86-90, Apr. 2000.

[4] LHCb Level-1 Vertex Topology Trigger, LHCb Tech. Note 99-31, CERN LHCb-98-069, Aug. 1999.

[5] Readout Unit, FPGA versión for multiplexers, DAQ and VELO trigger $\mathrm{LHCb}$ technical note in prep. 2001-xxx URL http://hmuller.home.cern.ch/hmuller/RU/LHCbNote.pdf

[6] J.Toledo, F.Bal, D.Domínguez,A.Guirao,H. Müller, "Readout Unit for high rate application", Proc. $12^{\text {th }}$ IEEE NPSS Real Time Conference, Valencia pp. 230-234, June 2001.

[7] A.Walsch, V.Lindenstruth, M. Schulz "Evaluation of SCI as a fabric for a computer based pattern recognition trigger running at 1.12 MHz", Proc. $12^{\text {th }}$ IEEE NPSS Real Time Conference, Valencia pp. 11-15, June 2001

[8] Scalable Coherent Interface (SCI), IEEE-1596 1982 standard, an example of commercial SCI: URL http://www.dolphinics.com

[9] J. Tole, H. Müller, F. Bal, B. Jost, "Readout Unit for the $\mathrm{LHCb}$ experiment" Proc. Fifth Workshop on Electronics for LHC experiments, Snowmass, Colorado, pp. 352-356, . Sept.1999.

[10] A.Guirao, J. Toledo, D. Domínguez, B. Bruder, H. Müller, "A networked mezzanine card processor" , Proc. $12^{\text {th }}$ IEEE NPSS Real Time Conference, Valencia 2001, pp. 81-84, June 2001.

[11] Detector Control System for an LHC experiment, P.Mato et al.. CERN LHCb-98-005 